\title{
Corrigendum: The genomic landscape of juvenile myelomonocytic leukemia
}

Elliot Stieglitz, Amaro N Taylor-Weiner, Tiffany Y Chang, Laura C Gelston, Yong-Dong Wang, Tali Mazor, Emilio Esquivel, Ariel Yu, Sara Seepo, Scott R Olsen, Mara Rosenberg, Sophie L Archambeault, Ghada Abusin, Kyle Beckman, Patrick A Brown, Michael Briones, Benjamin Carcamo, Todd Cooper, Gary V Dahl, Peter D Emanuel, Mark N Fluchel, Rakesh K Goyal, Robert J Hayashi, Johann Hitzler, Christopher Hugge, Y Lucy Liu, Yoav H Messinger, Donald H Mahoney Jr, Philip Monteleone, Eneida R Nemecek, Philip A Roehrs, Reuven J Schore, Kimo C Stine, Clifford M Takemoto, Jeffrey A Toretsky, Joseph F Costello, Adam B Olshen, Chip Stewart, Yongjin Li, Jing Ma, Robert B Gerbing, Todd A Alonzo, Gad Getz, Tanja A Gruber, Todd R Golub, Kimberly Stegmaier \& Mignon L Loh Nat. Genet. 47, 1326-1333 (2015); published online 12 October 2015; corrected after print 7 December 2015

In the version of this article initially published, two patients were stated on page 5 to have been excluded owing to insufficient follow-up data. These patients were included in the final analysis, but two additional patients were excluded owing to the presence of Noonan syndrome. On page 6, monosomy 7 was incorrectly listed as a significant factor in event-free and overall survival, but this factor was no longer significant after removing the patients with Noonan syndrome. The Online Methods incorrectly referred to "Data from patient AAML0122" instead of data from patients enrolled on AAML0122. The errors have been corrected in the HTML and PDF versions of the article.

\section{Corrigendum: Common variants at 19p13 are associated with susceptibility to ovarian cancer}

Kelly L Bolton, Jonathan Tyrer, Honglin Song, Susan J Ramus, Maria Notaridou, Chris Jones, Tanya Sher, Aleksandra Gentry-Maharaj, Eva Wozniak, Ya-Yu Tsai, Joanne Weidhaas, Daniel Paik, David J Van Den Berg, Daniel O Stram, Celeste Leigh Pearce, Anna H Wu, Wendy Brewster, Hoda Anton-Culver, Argyrios Ziogas, Steven A Narod, Douglas A Levine, Stanley B Kaye, Robert Brown, Jim Paul, James Flanagan, Weiva Sieh, Valerie McGuire, Alice S Whittemore, Ian Campbell, Martin E Gore, Jolanta Lissowska, Hanna P Yang, Krzysztof Medrek, Jacek Gronwald, Jan Lubinski, Anna Jakubowska, Nhu D Le, Linda S Cook, Linda E Kelemen, Angela Brooks-Wilson, Leon F A G Massuger, Lambertus A Kiemeney, Katja K H Aben, Anne M van Altena, Richard Houlston, Ian Tomlinson, Rachel T Palmieri, Patricia G Moorman, Joellen Schildkraut, Edwin S Iversen, Catherine Phelan, Robert A Vierkant, Julie M Cunningham, Ellen L Goode, Brooke L Fridley, Susan Kruger-Kjaer, Jan Blaeker, Estrid Hogdall, Claus Hogdall, Jenny Gross, Beth Y Karlan, Roberta B Ness, Robert P Edwards, Kunle Odunsi, Kirsten B Moyisch, Julie A Baker, Francesmary Modugno, Tuomas Heikkinenen, Ralf Butzow, Heli Nevanlinna, Arto Leminen, Natalia Bogdanova, Natalia Antonenkova, Thilo Doerk, Peter Hillemanns, Matthias Dürst, Ingo Runnebaum, Pamela J Thompson, Michael E Carney, Marc T Goodman, Galina Lurie, Shan Wang-Gohrke, Rebecca Hein, Jenny Chang-Claude, Mary Anne Rossing, Kara L Cushing-Haugen, Jennifer Doherty, Chu Chen, Thorunn Rafnar, Soren Besenbacher, Patrick Sulem, Kari Stefansson, Michael J Birrer, Kathryn L Terry, Dena Hernandez, Daniel W Cramer, Ignace Vergote, Frederic Amant, Diether Lambrechts, Evelyn Despierre, Peter A Fasching, Matthias W Beckmann, Falk C Thiel, Arif B Ekici, Xiaoqing Chen, the Australian Ovarian Cancer Study Group, the Australian Cancer Study (Ovarian Cancer), on behalf of the Ovarian Cancer Association Consortium, Sharon E Johnatty, Penelope M Webb, Jonathan Beesley, Stephen Chanock, Montserrat Garcia-Closas, Tom Sellers, Douglas F Easton, Andrew Berchuck, Georgia Chenevix-Trench, Paul D P Pharoah \& Simon A Gayther Nat. Genet. 42, 880-884 (2010); published online 19 September 2010; corrected after print 7 December 2015

In the version of this article initially published, the name of author Angela Brooks-Wilson was spelled incorrectly in the author list. The error has been corrected in the HTML and PDF versions of the article.

\section{Erratum: Large-scale identification of sequence variants influencing human transcription factor occupancy in vivo}

Matthew T Maurano, Eric Haugen, Richard Sandstrom, Jeff Vierstra, Anthony Shafer, Rajinder Kaul \& John A Stamatoyannopoulos Nat. Genet.; doi:10.1038/ng.3432; corrected online 17 November 2015

In the version of this article initially published online, the Online Methods incorrectly abbreviated mapping quality as MAQ rather than MAPQ. Also in the Online Methods, the procedure for downsampling allele counts for cross-cell type analysis of imbalance was incorrectly written as "we subsampled each site to three cell types and further downsampled the allele counts to mapping quality for the lowest of the three cell types." The sentence should read "we subsampled each site to three cell types and further downsampled to the allele counts to match the lowest of the three cell types.” The errors have been corrected for the print, PDF and HTML versions of this article. 